\title{
Utilização do sistema modular na prótese total da articulação coxofemoral em cães
}

\author{
Modular total hip replacement system in dogs \\ Bruno Watanabe Minto', Cláudia Valéria Seullner Brandão', \\ Gilberto José Cação Pereira² \& José Joaquim Titton Ranzani'
}

\begin{abstract}
RESUMO
A prótese total da articulação coxofemoral consiste na substituição completa da articulação por componentes protéticos acetabular e femoral. Esse procedimento representa uma das técnicas mais eficientes para o tratamento cirúrgico da displasia coxofemoral severa em cães. No presente relato foi experimentalmente aplicada uma prótese total da articulação coxofemoral de fabricação nacional em dois cães. Foi utilizada uma prótese com o sistema modular, ou seja, componente femoral provido de um sistema de cambiamento da cabeça femoral, com o objetivo de estabelecer a sua aplicabilidade, vantagens e avaliar a evolução pós-operatória. A prótese total modular da articulação coxofemoral foi aplicada em dois animais, da espécie canina (Canis familiaris), sadios, sem raça definida, e sem alterações ao aparelho locomotor. O procedimento foi realizado sem complicações e o resultado foi satisfatório em ambos os animais. A prótese total da articulação coxofemoral utilizando o sistema modular pode ser aplicada com sucesso em cães, desde que se tenha conhecimento prévio da técnica de aplicação, treinamento, e material específico. A principal vantagem da aplicação do sistema modular é a flexibilidade fornecida por este sistema, o qual permite escolher entre diferentes tamanhos de cabeça e comprimentos do colo femoral.
\end{abstract}

Descritores: cão, prótese total coxofemoral, sistema modular.

\section{ABSTRACT}

Total hip prosthesis is a full replacement of the hip by femoral and acetabular components. This is one of the most efficient surgical treatment procedures for severe canine dysplasia. In this work a hip replacement has been performed in two dogs using a prosthesis made in Brazil. A prosthesis having a modular system, i.e., the femoral component being supplied by an exchangeable femoral head system was used. The utilization of this procedure and postoperative outcomes were evaluated. The modular total hip prosthesis was applied in two healthy mongrel dogs (Canis familiaris), without orthopaedic alterations. The procedure was performed without complications and the results were satisfactory in both dogs. The modular total hip replacement can be performed successfully in dogs, provided that one has previous knowledge, training and proper equipment. The main modular system advantage is its versatility, allowing the choice among different head sizes and neck length.

Key words: $\operatorname{dog}$, total hip replacement, modular system. 


\section{INTRODUÇÃO}

A prótese total da articulação coxofemoral representa uma das técnicas mais indicadas para o tratamento cirúrgico da displasia coxofemoral canina nos Estados Unidos [16]. No Brasil, a técnica ainda é pouco difundida, principalmente por se tratar de um procedimento que exige treinamento especializado e importação dos componentes protéticos, tornando-o oneroso [3].

A displasia coxofemoral refere-se a um desenvolvimento anormal da articulação, caracterizada principalmente por frouxidão articular [7]. É a principal afecção causadora de doença articular degenerativa em cães [7,14,15]. Dentre os inúmeros tratamentos descritos para esta afecção a prótese total apresenta a vantagem de ser um procedimento que não somente preserva a função do membro como restabelece mecanismos articulares sem dor $[10,17,18]$.

Desde os meados dos anos 70, o procedimento protético da articulação coxofemoral canina tem sido realizado com sucesso [2,10,17], e consiste no implante de uma cúpula acetabular de polietileno de alta densidade e um componente femoral composto de cabeça e haste femoral de aço inoxidável, titânio, ou liga de cromo-cobalto $[4,12,17]$. Vários modelos protéticos têm sido descritos $[8,14,17]$; os primeiros desenvolvidos em Medicina Veterinária baseavam-se no sistema de cabeça femoral fixa [17]. Entretanto, este sistema mostrase pouco versátil, pois não há a possibilidade de cambiamento da cabeça femoral e alteração do comprimento do colo femoral $[11,14,17]$.

O presente trabalho teve por objetivos avaliar por meio de estudos clínicos e radiográficos a aplicação experimental de próteses totais coxofemorais nacionais utilizando o sistema modular.

\section{MATERIAIS E MÉTODOS}

Foi realizado o procedimento de prótese total modular da articulação coxofemoral em dois cães sem alterações articulares, sem raça definida, pesando 23 e $20 \mathrm{~kg}$. Os cães foram selecionados após exame clínico completo, com atenção especial aos exames ortopédico e neurológico. Os animais foram anestesiados utilizando-se anestesia epidural, e submetidos ao procedimento de prótese total da articulação coxofemoral esquerda. Realizou-se uma abordagem crânio-lateral da articulação com tenotomia parcial do músculo glúteo profundo [13]. Após osteotomia da cabeça e colo femorais, os leitos acetabular e femoral foram devidamente preparados para receber os componentes protéticos. Um componente acetabular de polietileno de alta densidade e uma haste femoral de cromo-cobalto provida de um sistema de cambiamento da cabeça femoral foram cimentados aos leitos previamente preparados. Após secagem do cimento, a articulação foi reduzida, testada e a cápsula articular suturada. Os tecidos moles adjacentes foram suturados com pontos simples separados, reaproximando-se os planos anatômicos. Após o procedimento cirúrgico os cães foram mantidos em repouso durante 10 dias, em jaulas individuais para limitar sua movimentação.

Para avaliação pós-operatória, os animais foram submetidos ao exame clínico e ortopédico, sempre pelo mesmo examinador, 24 horas após a cirurgia (Momento 1 - M1), 7 dias após a cirurgia (M2), 30 dias após a cirurgia (M3), 60 dias após a cirurgia (M4), e 120 dias após a cirurgia (M5). Estes exames objetivaram determinar, nos diferentes momentos de avaliação, a qualidade da locomoção, sustentação de peso sobre o membro operado, presença de dor ou aumento de volume, e alterações sistêmicas relacionadas. Exames radiográficos foram realizados nos mesmos momentos de avaliação, com exceção do M2.

\section{RESULTADOS}

A técnica de prótese total da articulação coxofemoral utilizando o sistema modular foi realizada com sucesso em ambos os cães. O material protético mostrou-se adequado e permitiu a realização de todas as etapas da técnica cirúrgica sem a ocorrência de complicações.

Os cães submetidos ao procedimento de prótese total da articulação coxofemoral apresentaram função locomotora satisfatória já no primeiro momento de avaliação, apresentando sustentação de peso no membro operado, entretanto com claudicação moderada. A função locomotora destes animais apresentou melhora progressiva com a evolução do pós-operatório, e a partir do sexagésimo dia foram registrados ausência de alterações na locomoção e dor na palpação das articulações operadas, o que representou resultado excelente na avaliação clínico-ortopédica.

Radiograficamente, os cães apresentaram posicionamento adequado dos componentes protéticos na avaliação pós-operatória imediata, observando-se encaixe desses componentes e redução articular. A cimentação pôde ser avaliada pela presença da fina 
camada de cimento ósseo ao redor dos componentes protéticos. A partir dos 30 dias, notou-se a presença de uma linha radiolucente de espessura inferior a $1 \mathrm{~mm}$ na interface entre a superfície óssea e o cimento ósseo na região acetabular.

\section{DISCUSSÃO}

A prótese total da articulação coxofemoral é amplamente utilizada para o tratamento da osteoartrite sintomática canina grave há mais de 30 anos [5,6]. Pelo alto custo da importação dos componentes protéticos e por se tratar de um procedimento que exige treinamento especializado, esta técnica é pouco estudada e também pouco realizada no Brasil. O desenvolvimento de um produto protético nacional permitiria uma maior disponibilidade de material cirúrgico e eliminaria, em parte, a limitação financeira ocasionada pela aplicação desta importante opção terapêutica. Além disto, o estudo continuado e sistemático da sua aplicação possibilitaria a capacitação e o aprimoramento dessa técnica na Medicina Veterinária brasileira. O desenvolvimento e a difusão deste importante procedimento são esperados, haja vista os excelentes resultados obtidos [14], portanto o material nacional certamente contribuirá muito com essa expectativa.

O procedimento de prótese total da articulação coxofemoral utilizando-se o sistema modular com cabeça femoral intercambiável mostrou-se efetivamente aplicável em cães hígidos. A recuperação dos animais foi rápida, com retorno da função normal do membro operado dentro de 60 dias. Estes dados condizem com os descritos na literatura [11]. O sistema modular de prótese total da articulação coxofemoral mostrouse flexível podendo adaptar-se as dimensões do material disponível às diferenças anatômicas de cada um dos animais. Esta é a principal vantagem deste sistema sobre as próteses totais da articulação coxofemoral que utilizam o sistema de cabeça femoral fixa, dados estes, descritos anteriormente $[1,11,14,17]$.

$\mathrm{Na}$ análise das radiografias verificou-se a presença de cimentação e encaixe adequado dos componentes acetabular e femoral (Figura 1), semelhante às descritas na literatura [9].

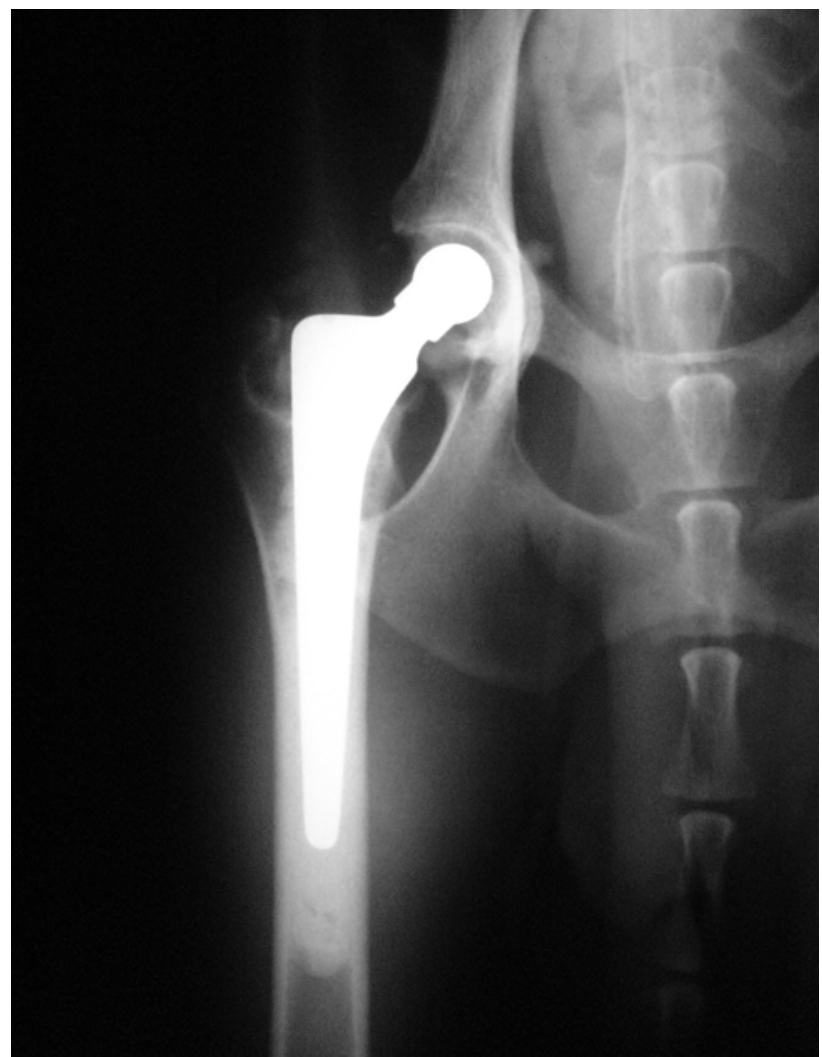

Figura 1. Imagem radiográfica ventrodorsal do cão submetido ao procedimento de prótese total da articulação coxofemoral esquerda, aos 60 dias do pós-operatório. Notar o posicionamento adequado dos componentes protéticos da articulação coxofemoral e o envolvimento dos mesmos pelo cimento ósseo [Foto: Bruno Watanabe Minto].

Apesar do número reduzido de animais submetidos ao procedimento e o curto período de avaliação, os resultados obtidos estimulam a continuidade do trabalho para determinação dos mesmos com um período de avaliação pós-operatória mais prolongado.

\section{CONCLUSÕES}

Esta técnica mostrou-se apta a ser testada em animais portadores de quadros graves de displasia coxofemoral no Brasil, desde que se tenha treinamento, equipamento e infra-estrutura adequados, além de conhecimento específico da técnica de aplicação.

Comissão de Ética: O presente trabalho foi aprovado pela Câmara de Ética em Experimentação Animal da FMVZ, Unesp, Campus de Botucatu.

\section{REFERÊNCIAS}

1 Arias S.A., Rezende C.M.F., Alvarez A. \& Souza M.V. 2004. Prótese total da articulação coxofemoral em cães: Relato de dois casos. Arquivo Brasileiro de Medicina Veterinária e Zootecnia. 56: 618-622. 
2 Bergh M.S., Muir P., Markel M.D. \& Manley P.A. 2004. Femoral bone adaptation to unstable long-term cemented total hip arthroplasty in dogs. Veterinary Surgery. 33: 238-245.

3 Brandão C.V.S., Pereira G.J.C., Minto B.W., Mamprim M.J., Dinhanne D.I. \& Ranzani J.J.T. 2003. Prótese total da articulação coxofemoral em cães: Relato de dois casos. In: Resumos do XXX Congresso Brasileiro de Medicina Veterinária (Manaus, Brasil). p.90.

4 De young D.J., De young B.A., Aberman H.A., Kenna R.V. \& Hungerford D.S. 1992. Implantation of uncemented total hip prosthesis. Technique and initial results of 100 arthroplasties. Veterinary Surgery. 21: 168-177.

5 Dearmin M.G. \& Schulz K.S. 2004. The effect of stem length on femoral component positioning in canine total hip arthroplasty. Veterinary Surgery. 33: 272-78.

6 Harasen G. 2005. Orthopedics odds and ends. Canadian Veterinary Journal. 46: 170-171.

7 Johnson A.L. \& Hulse D.A. 2002. Diseases of the joints. In: Fossum T.W. (Ed). Small Animal Surgery. 2nd edn. St. Louis: Mosby, pp.1023-1157.

8 Jones D.G.C. 1994. The hip joint. In: Houlton J \& Collinson R. (Ed.). Manual of Small Animal Arthrology. Bournemouth: Grafos S.A., pp.243-266.

9 Konde L.J., Olmstead M. \& Hohn R.B. 1982. Radiographic evaluation of total hip replacement in the dog. Veterinary Radiology and Ultrasound. 23: 98-106.

10 Olmstead M.L. 1987. Total hip replacement. Veterinary Clinics of North America: Small Animal Practice. 17: 943-954. 